Bull. Korean Math. Soc. 49 (2012), No. 4, pp. 715-736

http://dx.doi.org/10.4134/BKMS.2012.49.4.715

\title{
PERFECT IDEALS OF GRADE THREE DEFINED BY SKEW-SYMMETRIZABLE MATRICES
}

\author{
Yong Sung Cho, Oh-Jin Kang, And Hyoung June Ko*
}

\begin{abstract}
Brown provided a structure theorem for a class of perfect ideals of grade 3 with type 2 and $\lambda>0$. We introduced a skew-symmetrizable matrix to describe a structure theorem for complete intersections of grade 4 in a Noetherian local ring. We construct a class of perfect ideals $I$ of grade 3 with type 2 defined by a certain skew-symmetrizable matrix. We present the Hilbert function of the standard $k$-algebras $R / I$, where $R$ is the polynomial ring $R=k\left[v_{0}, v_{1}, \ldots, v_{m}\right]$ over a field $k$ with indeterminates $v_{i}$ and $\operatorname{deg} v_{i}=1$.
\end{abstract}

\section{Introduction}

Let $R$ be a commutative Noetherian local ring with maximal ideal $\mathfrak{m}$, and let $I$ be a proper ideal of $R$ with finite projective dimension. The type of a perfect ideal $I$ of grade $g$ is defined to be the dimension of $R / \mathfrak{m}$-vector space $\operatorname{Ext}_{R}^{g}(R / \mathfrak{m}, R / I)$. We denote it by type $I$. Equivalently, if

$$
\mathbb{F}: 0 \longrightarrow F_{g} \longrightarrow F_{g-1} \longrightarrow \cdots \longrightarrow F_{1} \longrightarrow R
$$

is the minimal free resolution of $R / I$, then type $I=\operatorname{rank} F_{g}$. A perfect ideal $I$ of grade $g$ is a complete intersection if $I$ is generated by a regular sequence $x_{1}, x_{2}, \ldots, x_{g}$, an almost complete intersection if it is minimally generated by $g+1$ elements, and a Gorenstein ideal if $I$ has type 1 .

In 1977, Buchsbaum and Eisenbud [4] provided structure theorems for Gorenstein ideals of grade 3 and for almost complete intersections of grade 3 . They also showed that every perfect ideal of grade 3 has a differential, graded commutative algebra structure. In 1987, Brown [2] provided a structure theorem for a class of perfect ideals of grade 3 with type 2 and $\lambda(I)>0$, where $\lambda(I)$ is the numerical invariant introduced by Kustin and Miller [12] to distinguish classes of Gorenstein ideals $I$ of grade 4 in terms of free resolutions of $R / I$. In 1989, Sanchez [14] provided a structure theorem for a class of type 3 perfect

Received March 26, 2011.

2010 Mathematics Subject Classification. 13C05, 13H10, 13C02, $13 \mathrm{C} 40$.

Key words and phrases. almost complete intersection of grade 3, perfect ideal of grade 3 , minimal free resolution, linkage.

* This work was supported by the Yonsei University Research Fund of 2012 Global Yonsei. 
ideals of grade 3 and $\lambda(I) \geq 2$. In 2005, Kang and Ko [9] provided a structure theorem for complete intersections of grade 4. In 2009, Kang, Cho, and Ko [8] provided a structure theorem for some classes of perfect ideals of grade 3 which are algebraically linked to an almost complete intersection of grade 3 by a regular sequence. This contains not only three classes of perfect ideals of grade 3 which are known by Buchsbaum and Eisenbud, Brown, and Sanchez, but also a class of perfect ideals of grade 3 with type 4 which are algebraically linked to an almost complete intersection of grade 3 by a regular sequence.

In $[5,10]$ we introduced some skew-symmetrizable matrices $G_{i}(i=1,2)$ and the ideals associated with $G_{i}$. We gave classes of ideals given by the quotient of the submaximal order Pfaffians of the alternating matrix induced by $G_{i} . G_{1}$ and $G_{2}$ define classes of perfect ideals of grade 3 with type $2, \lambda(I)>0$ and type $3, \lambda(I) \geq 2$, respectively. These perfect ideals are minimally generated by $n$ elements which are algebraically linked to almost complete intersections of grade 3 with even type by a regular sequence, where $n$ is odd and $n>3$.

This shows that the skew-symmetrizable matrices and the ideals associated with them play a key role in distinguishing some classes of perfect ideals of grade 3 with type $2 \leq r \leq 4$ minimally generated by $n$ elements, where $n$ is odd and $n>3$. In this paper, we define a certain skew-symmetrizable matrix $G_{3}$ determined by an $r \times 4$ matrix $A$, an $r \times r$ alternating matrix $Y$ and a $4 \times 4$ alternating matrix $U$ [see (3.3)], and the ideal $\overline{P f_{r+3}\left(G_{3}\right)}$ associated with $G_{3}$. The main purpose of this paper is to construct a class of perfect ideals of grade 3 with type 2 , which are minimally generated by the quotients of the submaximal order Pfaffians of the alternating matrix induced by $G_{3}$. These ideals contain some perfect ideals of grade 3 with type 2 and $\lambda(I)=0$. Now we describe the contents of this paper.

In Section 2, we review linkage theory and some structure theorems for perfect ideals of grade 3 .

In Section 3, we provide useful properties of the skew-symmetrizable matrix $G_{i}(i=1,2)$ and define the ideal $I=\overline{P f_{*}\left(G_{i}\right)}$ associated with $G_{i}$. We introduce an other skew-symmetrizable matrix $G_{3}$ determined by $A, Y$ and $U$. We construct a class of perfect ideals $I$ of grade 3 with type 2 defined by $G_{3}$, and the minimal free resolution $\mathbb{F}$ of $R / I$.

In Section 4, we compute the Hilbert function of homogeneous perfect ideals $I$ of grade 3 with type 2 , which are minimally generated by the quotients of the submaximal order Pfaffians of the alternating matrix induced by a skewsymmetrizable matrix $G_{3}$.

\section{Preliminaries}

In this section, we review linkage theory and the structure theorems for three classes of perfect ideals of grade 3, which are given by Buchsbaum and Eisenbud, Brown, and Sanchez. To review these structure theorems, first we need some properties of an alternating matrix. Let $T=\left(t_{i j}\right)$ be an $n \times n$ 
alternating matrix with entries in a commutative ring $R$. It is well known that if $n$ is odd, the determinant of an alternating matrix $T$ is zero, and if $n$ is even, it is a square of a homogeneous polynomial of degree $\frac{n}{2}$ in the entries of $T$, which is called the pfaffian of $T$. We will write $\operatorname{det} T=\operatorname{Pf}(T)^{2}$. Denote by $P f_{s}(T)$ the ideal generated by the $s$ th order Pfaffians of $T$. Let $s<n$ and $(i)=i_{1}, i_{2}, \ldots, i_{s}$ denote an index of integers. Let $\theta(i)$ denote the sign of the permutation that rearranges $(i)$ in increasing order. If $(i)$ has a repeated index, then we set $\theta(i)=0$. Let $\tau(i)$ be the sum of the entries of $(i)$ and $T\left(i_{1}, i_{2}, \ldots, i_{s}\right)$ an alternating submatrix of $T$ formed by deleting rows and columns $i_{1}, i_{2}, \ldots, i_{s}$ from $T$. Define

$$
T_{(i)}=(-1)^{\tau(i)+1} \theta(i) \operatorname{Pf}\left(T\left(i_{1}, i_{2}, \ldots, i_{s}\right)\right) .
$$

If $s=n$, we let $T_{(i)}=(-1)^{\tau(i)+1} \theta(i)$ and if $s>n$, we let $T_{(i)}=0$. Let $\mathbf{t}=\left[\begin{array}{llll}T_{1} & T_{2} & \cdots & T_{n}\end{array}\right]$ be the row vector of the maximal order Pfaffians of $T$, signed appropriately according to the conventions described above. Pfaffians can be developed along a row in the same manner as the determinants. There is a "Laplace expansion" for developing Pfaffians in terms of those of lower order.

Lemma 2.1 ([Lemma 1.1, 12]). Let $T$ be an $n \times n$ alternating matrix and $j a$ fixed integer, $1 \leq j \leq n$. Then

(1) $\operatorname{Pf}(T)=\sum_{i=1}^{n} t_{i j} T_{i j}$, and

(2) $\mathbf{t} T=0$.

Lemma 2.2 follows from Lemma 2.1.

Lemma 2.2 ([Lemma 1.1, 14]). Let $T$ be an $n \times n$ alternating matrix. Let $a, b, c, d$, and $e$ be distinct integers between 1 and $n$. Then

(1) $\sum_{i=1}^{n} t_{i k} T_{i a b}=-\delta_{k a} T_{b}+\delta_{k b} T_{a}$

(2) $\sum_{i=1}^{n} t_{i k} T_{i a b c}=\delta_{k a} T_{b c}-\delta_{k b} T_{a c}+\delta_{k c} T_{a b}$,

(3) $\sum_{i=1}^{n} t_{i k} T_{i a b c d}=-\delta_{k a} T_{b c d}+\delta_{k b} T_{a c d}-\delta_{k c} T_{a b d}+\delta_{k d} T_{a b c}$, and

(4) $\sum_{i=1}^{n} t_{i k} T_{i a b c d e}=\delta_{k a} T_{b c d e}-\delta_{k b} T_{a c d e}+\delta_{k c} T_{a b d e}-\delta_{k d} T_{a b c e}+\delta_{k e} T_{a b c d}$, where $\delta_{i j}$ is the Kronecker's delta.

For further analysis, we have the following lemma from Lemmas 2.1 and 2.2.

Lemma 2.3 ([Corollary 2.1, 11]). Let $n$ be a positive integer and $T$ an $n \times n$ alternating matrix. Assume that $i, j, k$, and $l$ are elements of a set $\{1,2, \ldots, n\}$. 
Then

$$
T_{i} T_{j k l}-T_{j} T_{i k l}+T_{k} T_{i j l}-T_{l} T_{i j k}=0 .
$$

Let $F$ be a free $R$-module with $\operatorname{rank} F=n$. We denote by $F^{*}$ the dual module of $F$.

The Buchsbaum and Eisenbud structure theorem identifies Gorenstein ideals of grade 3 as the ideals $P f_{n-1}(T)=\left(T_{1}, T_{2}, \ldots, T_{n}\right)$ of a certain $n \times n$ alternating matrix $T$.

Theorem 2.4 ([Theorem 2.1,4]). Let $R$ be a Noetherian local ring with maximal ideal $\mathfrak{m}$.

(1) Let $n \geqslant 3$ be an odd integer. Let $F$ be a free $R$-module with $\operatorname{rank} F=n$. Let $f: F^{*} \rightarrow F$ be an alternating map whose image is contained in $\mathfrak{m} F$. Suppose that $P f_{n-1}(f)$ has grade 3. Then $P f_{n-1}(f)$ is a Gorenstein ideal minimally generated by $n$ elements.

(2) Every Gorenstein ideal of grade 3 arises as in this way.

We noticed that, as in [4] or [13], in most cases, linkage is used in the case of perfect ideals in Gorenstein or Cohen-Macaulay local rings. However the results we will use are true for perfect ideals in any commutative ring, as shown by Golod [6].

Definition 2.5. Let $I$ and $J$ be perfect ideals of grade $g$. An ideal $I$ is linked to $J, I \sim J$ if there exists a regular sequence $\mathbf{x}=x_{1}, x_{2}, \ldots, x_{g}$ in $I \cap J$ such that $J=(\mathbf{x}): I$ and $I=(\mathbf{x}): J$, and $I$ is geometrically linked to $\mathrm{J}$ if $I \sim J$ and $I \cap J=(\mathbf{x})$.

A fundamental result is that linkage is a symmetric relation on the set of perfect ideals in a Noetherian ring $R$.

Theorem 2.6 ([Proposition 1.3, 13]). Let $R$ be a Noetherian ring. If $I$ is a perfect ideal of grade $g$ and $\mathbf{x}=x_{1}, x_{2}, \ldots, x_{g}$ is a regular sequence in $I$, then $J=(\mathbf{x}): I$ is a perfect ideal of grade $g$ and $I=(\mathbf{x}): J$.

An almost complete intersection of grade $g$ is linked to a Gorenstein ideal of grade $g$ by a regular sequence $\mathbf{x}$.

Proposition 2.7 ([Proposition 5.2, 4]). Let $I$ and $J$ be perfect ideals of the same grade $g$ in a Noetherian local ring $R$ and suppose that $I$ is linked to $J$ by a regular sequence $\mathbf{x}=x_{1}, x_{2}, \ldots, x_{g}$. Then

(1) If $I$ is Gorenstein, then $J=(\mathbf{x}, w)$ for some $w \in R$ and

(2) If $J$ is minimally generated by $\mathbf{x}$ and $w$, then $I$ is Gorenstein.

Now we review the structure theorems for a class of perfect ideals $I$ of grade 3 with type $2, \lambda(I)>0$ and for a class of perfect ideals $I$ of grade 3 with type $3, \lambda(I) \geq 2$ given by Brown [2] and Sanchez [14]. Kustin and Miller introduced the numerical invariant $\lambda(I)$ defined in [12] to distinguish Gorenstein ideals $I$ of grade 4 in terms of a resolution of $R / I$. Brown provided a structure theorem 
for a class of perfect ideals $I$ of grade 3 with type $2, \lambda(I)>0$. The minimal free resolution $\mathbb{F}$ of $R / I$ is described in [2].

Theorem 2.8 ([Theorem 4.4, 2]). Let $R$ be a Noetherian local ring with maximal ideal $\mathfrak{m}$. Let $n>4$ be an integer. Let I be a type 2 perfect ideal of grade 3 minimally generated by $n$ elements. If $\lambda(I)>0$, then there is an $n \times n$ alternating matrix $T=\left(t_{i j}\right)$ with $t_{12}=0$ and $t_{i j}$ in $\mathfrak{m}$ such that

(1) if $n$ is odd, then $I=\left(T_{1}, T_{2}, z_{1} T_{12 j}+z_{2} T_{j}: 3 \leq j \leq n\right)$ for some $z_{1}, z_{2}$ in $\mathfrak{m}$,

(2) if $n$ is even, then $I=\left(P f(T), T_{12}, z_{1} T_{1 j}+z_{2} T_{2 j}: 3 \leq j \leq n\right)$ for some $z_{1}, z_{2}$ in $\mathfrak{m}$.

Sanchez provided a structure theorem for a class of perfect ideals $I$ of grade 3 with type $3, \lambda(I) \geq 2$. The minimal free resolution $\mathbb{F}$ of $R / I$ is described in [14].

Theorem 2.9 ([Theorem 2.1, 14]). Let $R$ be a Noetherian local ring with maximal ideal $\mathfrak{m}$. Let $I$ be a perfect ideal of grade 3 minimally generated by $n>4$ elements. If $I$ has type 3 and $\lambda(I) \geq 2$, then there exists an $n \times n$ alternating matrix $T=\left(t_{i j}\right)$ and a $2 \times 3$ matrix $X=\left(x_{i j}\right)$ with $t_{i j}, x_{i j}$ in $\mathfrak{m}$ such that

(1) if $n>3$ is odd, then either

$$
\begin{aligned}
I= & \left(T_{1}, x_{11} T_{2}+x_{12} T_{3}+x_{13} T_{123}, x_{21} T_{2}+x_{22} T_{3}+x_{23} T_{123},\right. \\
& \left.\Delta_{3} T_{j}+\Delta_{2} T_{12 j}+\Delta_{1} T_{13 j}: 4 \leq j \leq n\right)
\end{aligned}
$$

or

$$
\begin{aligned}
I= & \left(T_{123}, x_{11} T_{1}+x_{12} T_{2}+x_{13} T_{3}, x_{21} T_{1}+x_{22} T_{2}+x_{23} T_{3},\right. \\
& \left.\Delta_{3} T_{12 j}+\Delta_{2} T_{13 j}+\Delta_{1} T_{23 j}: 4 \leq j \leq n\right),
\end{aligned}
$$

where $\Delta_{i}$ is the determinant of the $2 \times 2$ submatrix of $X$ obtained by deleting the ith column;

(2) if $n>3$ is even, then either

$$
\begin{aligned}
I= & \left(P f(T), x_{11} T_{12}+x_{12} T_{13}+x_{13} T_{23}, x_{21} T_{12}+x_{22} T_{13}+x_{23} T_{23},\right. \\
& \left.\Delta_{3} T_{1 j}+\Delta_{2} T_{2 j}+\Delta_{1} T_{123 j}: 4 \leq j \leq n\right)
\end{aligned}
$$

or

$$
\begin{aligned}
I= & \left(T_{12}, x_{11} P f(T)+x_{12} T_{13}+x_{13} T_{23}, x_{21} \operatorname{Pf}(T)+x_{22} T_{13}+x_{23} T_{23},\right. \\
& \left.\Delta_{3} T_{1 j}+\Delta_{2} T_{2 j}+\Delta_{1} T_{123 j}: 4 \leq j \leq n\right) .
\end{aligned}
$$

We introduced an almost complete matrix $f$ of grade 3 determined by an $r \times 3$ matrix $A$ and an $r \times r$ alternating matrix $Y$, the extracted matrix from $f$, and an ideal $\mathcal{K}_{3}(f)$ associated with $f$ to give the structure theorem and characterizations of almost complete intersections of grade 3 with type $r$ [see Theorems 3.2 and 4.8, and Definitions 3.3 and 3.5, 8]. First we show that if $\mathcal{K}_{3}(f)$ has grade 3 , then the minimal free resolution $\mathbb{F}$ of $R / \mathcal{K}_{3}(f)$ has the following form. 
Theorem 2.10 ([Theorem 4.1, 8]). Let $A, Y, C, E, F, S, Z, \mathbf{z}, w$ be notations defined in Section 3 of [8] over the Noetherian local ring $R$ with maximal ideal $\mathfrak{m}$. Let $f$ be an almost complete matrix of grade 3 determined by $A$ and $Y$. Let $\widetilde{f}$ be a $4 \times(r+3)$ matrix extracted from $f$.

(1) If $r$ is even and if $\mathcal{K}_{3}(f)$ has grade 3 , then a minimal free resolution of $R / \mathcal{K}_{3}(f)$ has the form:

$$
\mathbb{F}: 0 \longrightarrow R^{r} \stackrel{f_{3}}{\longrightarrow} R^{r+3} \stackrel{f_{2}}{\longrightarrow} R^{4} \stackrel{f_{1}}{\longrightarrow} R,
$$

where

$$
f_{1}=\left[\begin{array}{ll}
C & w
\end{array}\right], f_{2}=\tilde{f}=\left[\begin{array}{c|c}
Z & S \\
\hline C & E
\end{array}\right], f_{3}=\left[\begin{array}{c}
F \\
Y
\end{array}\right] .
$$

(2) If $r$ is odd and if $\mathcal{K}_{3}(f)$ has grade 3 , then a minimal free resolution of $R / \mathcal{K}_{3}(f)$ has the form:

$$
\mathbb{F}: 0 \longrightarrow R^{r} \stackrel{f_{3}}{\longrightarrow} R^{3+r} \stackrel{f_{2}}{\longrightarrow} R^{4} \stackrel{f_{1}}{\longrightarrow} R,
$$

where

$$
f_{1}=\left[\begin{array}{ll}
\mathbf{z} & w
\end{array}\right], \quad f_{2}=\tilde{f}=\left[\begin{array}{c|c}
Z & S \\
\hline C & E
\end{array}\right], \quad f_{3}=\left[\begin{array}{ll}
A & Y
\end{array}\right]^{t} .
$$

We also described the structure theorem for some classes of perfect ideals of grade 3 that are algebraically linked to an almost complete intersection of grade 3 by a regular sequence $\mathbf{x}=x_{1}, x_{2}, x_{3}$.

Theorem 2.11 ([Theorem 5.5, 8]). Let $R$ be a Noetherian local ring with maximal ideal $\mathfrak{m}$.

(1) Let $J$ be an almost complete intersection of grade 3 and let $B$ a matrix defined in (5.1) of [8]. Let $\mathbf{x}=x_{1}, x_{2}, x_{3}$ be a regular sequence in $J$ defined in (5.1) of [8]. Let $r$ be the type of $J$.

(i) Let $r$ be even. Let $A, E, S$, and $Y$ be matrices defined in (3.2), (3.3) of [8] and $p_{k 1}$ an element defined in (5.3) of [8] for $k=1,2, \ldots, r$.

(ii) Let $r$ be odd. Let $A, S, Y$, and $Z$ be matrices defined in (3.3), (3.4) of [8] and $p_{k 1}$ an element defined in (5.4) of [8] for $k=1,2, \ldots, r$.

If $I$ is an ideal generated by $x_{1}, x_{2}, x_{3}, p_{11}, p_{21}, \ldots, p_{r 1}$, then $I$ is a perfect ideal of grade 3 linked to $J$ by a regular sequence $\mathbf{x}$ and has type $\mu(J /(\mathbf{x}))$.

(2) Every perfect ideal of grade 3 linked to an almost complete intersection $J$ of grade 3 by a regular sequence $\mathbf{x}=x_{1}, x_{2}, x_{3}$ arises as in the way of (1).

The structure theorems for some classes of perfect ideals of grade 3 determined by Buchsbaum and Eisenbud, Brown, and Sanchez can be regarded as a special case of Theorem 2.11. 


\section{Perfect ideals defined by skew-symmetrizable matrices}

We introduced a skew-symmetrizable matrix in [9] to define a complete matrix of grade 4 which plays a key role in describing a structure theorem for complete intersections of grade 4 . In this section, we study the ideal $I$ associated with a skew-symmetrizable matrix $G_{3}$ of grade 3 determined by an $r \times 4$ matrix $A$ and an $r \times r$ alternating matrix $Y$ and a $4 \times 4$ alternating matrix $U$ with entries in the maximal ideal $\mathfrak{m}$ of a Noetherian local ring $R$. We begin this section with the definition of a skew-symmetrizable matrix.

Definition 3.1. Let $R$ be a commutative ring with identity. An $n \times n$ matrix $X$ over $R$ is said to be skew-symmetrizable or generalized alternating if there exist nonzero diagonal matrices $D^{\prime}=\operatorname{diag}\left\{u_{1}, u_{2}, \ldots, u_{n}\right\}$ and $D=$ $\operatorname{diag}\left\{w_{1}, w_{2}, \ldots, w_{n}\right\}$ with entries in $R$ such that $D^{\prime} X D$ is an alternating matrix.

Let $X$ be an $n \times n$ skew-symmetrizable matrix. Then $\tilde{X}=D^{\prime} X D$ is an alternating matrix for some diagonal matrices $D^{\prime}$ and $D$. We set $\mathcal{A}(X)$ to be an alternating matrix given by

$$
\mathcal{A}(X)= \begin{cases}X & \text { if } X \text { is alternating, } \\ \tilde{X} & \text { if } X \text { is not alternating. }\end{cases}
$$

Example 3.2. Let $R$ be a commutative Noetherian local ring with maximal ideal $\mathfrak{m}$, and let $n$ be an odd integer with $n>3$. Let $Y=\left(y_{i j}\right)$ be an $n \times n$ alternating matrix with $y_{12}=0$ and entries in $\mathfrak{m}$, and let $A$ be the submatrix of $Y$ obtained by deleting the first two columns and the last $(n-2)$ rows of $Y$. For two elements $v$ and $w$ in $\mathfrak{m}$, we define the $n \times n$ skew-symmetrizable matrix $G_{1}$ by

$$
G_{1}=\left[\begin{array}{c|c}
B & v A \\
\hline-A^{t} & Y(1,2)
\end{array}\right] \text {, where } B=\left[\begin{array}{cc}
0 & w \\
-w & 0
\end{array}\right]
$$

and $Y(1,2)$ is the $(n-2) \times(n-2)$ alternating submatrix $Y$ obtained by removing the first, second rows and columns from $Y$. The alternating matrix $\mathcal{A}\left(G_{1}\right)$ is obtained by multiplying the first two columns of $G_{1}$ by $v$. Let $x_{i}$ be an element defined by

$$
x_{i}=\mathcal{A}\left(G_{1}\right)_{i} / v \text { for } i=1,2,3, \ldots, n .
$$

We define $\overline{P f_{n-1}\left(G_{1}\right)}$ as the ideal generated by $n$ elements $x_{1}, x_{2}, \ldots, x_{n}$. The next theorem states that $\overline{P f_{n-1}\left(G_{1}\right)}$ characterizes a perfect ideal $I$ of grade 3 satisfying the following properties: (1) $I$ has type 2, (2) the number of generators for $I$ is odd, and (3) $\lambda(I)>0$.

Theorem 3.3 ([Theorem 4.3, 10]). Let $R$ be a commutative Noetherian local ring with maximal ideal $\mathfrak{m}$. Let $n$ be an odd integer with $n>3$ and $v, w$ elements in $\mathfrak{m}$. Let $G_{1}$ be the $n \times n$ skew-symmetrizable matrix as defined in (3.1). 
(1) If $I=\overline{P f_{n-1}\left(G_{1}\right)}$ is an ideal of grade 3 with $\lambda(I)>0$, then $I$ is a perfect ideal of type 2 .

(2) Every perfect ideal of grade 3 with type 2 and $\lambda(I)>0$ minimally generated by $n$ elements arises as in the way of (1).

Next we construct a skew-symmetrizable matrix which defines a class of perfect ideals of grade 3 with type $3, \lambda(I) \geq 2$.

Definition 3.4. Let $R$ be a commutative Noetherian local ring with maximal ideal $\mathfrak{m}$, and let $A=\left(a_{i j}\right)$ and $Y=\left(y_{i j}\right)$ be an $r \times 3$ matrix and an $r \times r$ alternating matrix with entries in $\mathfrak{m}$, respectively. Set $F$ to be the $3 \times r$ matrix defined by

$$
F=\left[\begin{array}{cccc}
a_{11} & a_{21} & \cdots & a_{r 1} \\
-a_{12} & -a_{22} & \cdots & -a_{r 2} \\
a_{13} & a_{23} & \cdots & a_{r 3}
\end{array}\right]
$$

where $r$ is an even integer and $r \geq 4$. Define an $(r+3) \times(r+3)$ skewsymmetrizable matrix $G_{2}$ by

$$
G_{2}=\left[\begin{array}{c|c}
\mathbf{0} & \bar{F} \\
\hline-F^{t} & Y
\end{array}\right] \text {, where } \bar{F}=\left[\begin{array}{cccc}
v a_{11} & v a_{21} & \cdots & v a_{r 1} \\
-u a_{12} & -u a_{22} & \cdots & -u a_{r 2} \\
u v a_{13} & u v a_{23} & \cdots & u v a_{r 3}
\end{array}\right]
$$

with $u, v \in \mathfrak{m} \backslash\{0\}$. The alternating matrix $\mathcal{A}\left(G_{2}\right)$ is obtained by multiplying the first column of $G_{2}$ by $v$, the second column by $u$, and the third column by $u v$. To describe some of the non-Gorenstein perfect ideals of grade 3 , we need the ideal $\overline{P f_{r+2}\left(G_{2}\right)}$ induced from the submaximal order Pfaffians of $\mathcal{A}\left(G_{2}\right)$ as follows. Let

$$
\mathcal{A}\left(G_{2}\right)=\left[\begin{array}{c|c}
\mathbf{0} & \bar{F} \\
\hline-\bar{F}^{t} & Y
\end{array}\right]
$$

be the alternating matrix induced by $G_{2}$. We define $\overline{P f_{r+2}\left(G_{2}\right)}$ to be the ideal generated by the quotients of the submaximal order Pfaffians of $\mathcal{A}\left(G_{2}\right)$ by $u v$,

$$
\overline{P f_{r+2}\left(G_{2}\right)}=\left(\mathcal{A}\left(G_{2}\right)_{1} / u v, \mathcal{A}\left(G_{2}\right)_{2} / u v, \ldots, \mathcal{A}\left(G_{2}\right)_{r+3} / u v\right) .
$$

Theorem 3.5 ([Theorem 3.6, 5]). Let $R$ be a commutative Noetherian local ring with maximal ideal $\mathfrak{m}$. Let $x_{1}=\mathcal{A}\left(G_{2}\right)_{1} / u v, x_{2}=\mathcal{A}\left(G_{2}\right)_{2} /$ uv, and $x_{3}=$ $\mathcal{A}\left(G_{2}\right)_{3} / u v$. If $\mathbf{x}=x_{1}, x_{2}, x_{3}$ is a regular sequence in $\overline{P f_{r+2}\left(G_{2}\right)}$, then (1) $(\mathbf{x}): \overline{P f_{r+2}\left(G_{2}\right)}$ is a type $r$, grade 3 almost complete intersection, and (2) $\overline{P f_{r+2}\left(G_{2}\right)}$ is a type 3 , grade 3 perfect ideal.

Now we introduce the other skew-symmetrizable matrix to define a class of type 2 perfect ideals of grade 3 . 
Definition 3.6. Let $S$ be a regular element in a commutative ring $R$ with identity, and let $r$ be an odd integer with $r>1$. Let $A=\left(a_{i j}\right), U=\left(u_{i j}\right)$ and $Y=\left(y_{i j}\right)$ be an $r \times 4$ matrix, a $4 \times 4$ alternating matrix, and an $r \times r$ alternating matrix, respectively. Now we define $G_{3}$ to be an $(r+4) \times(r+4)$ skew-symmetrizable matrix as follows:

$$
G_{3}=\left[\begin{array}{c|c}
U & s A^{t} \\
\hline-A & Y
\end{array}\right] .
$$

The following lemma gives us a property to define ideals associated with a skew-symmetrizable matrix $G_{3}$.

Lemma 3.7. Using the notation given above, let $G_{3 i}$ be the $(r+3) \times(r+3)$ submatrix of $G$ obtained by deleting the ith column and row from $G_{3}$.

(1) $\operatorname{det} G_{3 i}$ is divisible by $s$ for $i=1,2,3,4$.

(2) $s^{3} \operatorname{det} G_{3 i}=\mathcal{A}\left(G_{3}\right)_{i}^{2}$ for $i=1,2,3,4$.

(3) $s^{4} \operatorname{det} G_{3 i}=\mathcal{A}\left(G_{3}\right)_{i}^{2}$ for $i=5,6, \ldots, r+4$.

Proof. This follows from direct computations.

We need the following lemma for further use.

Lemma 3.8. Using the notation given above, let $T=\left(t_{i j}\right)=\mathcal{A}\left(G_{3}\right)$ be the $(r+4) \times(r+4)$ alternating matrix induced by a skew-symmetrizable matrix $G_{3}$.

(1) If $i, j, k$ are integers with $1 \leq i, j, k \leq 4$, then $T_{i j k}$ is divisible by $s$;

(2) If $i, j, k$ are integers with $1 \leq i, j \leq 4$ and $5 \leq k \leq r+4$, then $T_{i j k}$ is divisible by $s$;

(3) If $i, j, k$ are integers with $5 \leq i, j, k \leq r+4$, then $T_{i j k}$ is divisible by $s^{2}$;

(4) If $i, j, k$ are integers with $1 \leq i \leq 4$ and $5 \leq j, k \leq r+4$, then $T_{i j k}$ is divisible by $s^{2}$;

(5) $T_{i}$ is divisible by $s^{2}$ for every $i$.

Proof. The first four parts follow from direct computations and the last part follows from Lemma 3.7.

Notations 3.9. Using the notation given in Lemmas 3.7 and 3.8, we let $T=$ $\mathcal{A}\left(G_{3}\right)$. Then from Lemma 3.7 we define $\bar{T}_{i}$ as the element given by

$$
T_{i}=s^{2} \bar{T}_{i} .
$$

If $i, j, k$ are integers in parts (1) or (2) of Lemma 3.8, then we define $\bar{T}_{i j k}$ as the element given by

$$
T_{i j k}=s \bar{T}_{i j k}
$$

and if $i, j, k$ are integers in parts (3) or (4) of Lemma 3.8, then we define $\bar{T}_{i j k}$ as the element given by

$$
T_{i j k}=s^{2} \bar{T}_{i j k} .
$$


In this way, if $(i)$ is the multi-index $i_{1}, i_{2}, \ldots, i_{n}$ and $T_{(i)}$ is divisible by $s$ or $s^{2}$, then we define $\bar{T}_{(i)}$ as the element given by

$$
T_{(i)}=s \bar{T}_{(i)} \quad \text { or } \quad T_{(i)}=s^{2} \bar{T}_{(i)} .
$$

To avoid the confusion of multi indexes we will denote $T_{i-j k}$ by $T_{i-j, k}$. The following lemma is also a consequence of Lemmas 2.2, 3.7 and 3.8.

Lemma 3.10. Let $G_{3}$ be an $(r+4) \times(r+4)$ skew-symmetrizable matrix and $T=\mathcal{A}\left(G_{3}\right)$ be the alternating matrix induced by $G_{3}$. Let $a, b, c, d$, and $e$ be distinct integers between 1 and $r+4$.

(1) Let $k$ be an integer with $1 \leq k \leq 4$.

(a) If $1 \leq a, b \leq 4$, then we have

$$
\sum_{i=1}^{4} u_{i k} \bar{T}_{i a b}+\sum_{i=5}^{r+4}-a_{i-4, k} \bar{T}_{i a b}=-\delta_{k a} \bar{T}_{b}+\delta_{k b} \bar{T}_{a} .
$$

(b) If $1 \leq a \leq 4$ and $5 \leq b \leq r+4$ or $5 \leq a \leq r+4$ and $1 \leq b \leq 4$, then we have

$$
\sum_{i=1}^{4} u_{i k} \bar{T}_{i a b}+\sum_{i=5}^{r+4}-s a_{i-4, k} \bar{T}_{i a b}=-\delta_{k a} \bar{T}_{b}+\delta_{k b} \bar{T}_{a} .
$$

(2) Let $k$ be an integer with $5 \leq k \leq r+4$.

(a) If $1 \leq a \leq 4$ and $5 \leq b \leq r+4$ or $5 \leq a \leq r+4$ and $1 \leq b \leq 4$, then we have

$$
\sum_{i=1}^{4} a_{k-4, i} \bar{T}_{i a b}+\sum_{i=5}^{r+4} y_{i-4, k-4} \bar{T}_{i a b}=-\delta_{k a} \bar{T}_{b}+\delta_{k b} \bar{T}_{a} .
$$

(b) If $5 \leq a, b \leq r+4$, then we have

$$
\sum_{i=1}^{4} s a_{k-4, i} \bar{T}_{i a b}+\sum_{i=5}^{r+4} y_{i-4, k-4} \bar{T}_{i a b}=-\delta_{k a} \bar{T}_{b}+\delta_{k b} \bar{T}_{a} .
$$

The following corollary is a consequence of Lemma 3.10.

Corollary 3.11. Using the notation given in Lemma 3.10, we have the following:

(1) Let $k$ be an integer with $1 \leq k \leq 4$

(a) If $1 \leq a, b \leq 4$, then we have

$$
\sum_{i=1}^{4} u_{i k} \bar{T}_{i a b}+\sum_{i=5}^{r+4}-a_{i-4, k} \bar{T}_{i a b}=0 \quad \text { for } k \neq a, b .
$$

(b) If $1 \leq a \leq 4$ and $5 \leq b \leq r+4$ or $5 \leq a \leq r+4$ and $1 \leq b \leq 4$, then we have

$$
\sum_{i=1}^{4} u_{i k} \bar{T}_{i a b}+\sum_{i=5}^{r+4}-s a_{i-4, k} \bar{T}_{i a b}=0 \quad \text { for } k \neq a, b .
$$


(2) Let $k$ be an integer with $5 \leq k \leq r+4$.

(a) If $1 \leq a \leq 4$ and $5 \leq b \leq r+4$ or $5 \leq a \leq r+4$ and $1 \leq b \leq 4$, then we have

$$
\sum_{i=1}^{4} a_{k-4, i} \bar{T}_{i a b}+\sum_{i=5}^{r+4} y_{i-4, k-4} \bar{T}_{i a b}=0 \quad \text { for } k \neq a, b .
$$

(b) If $5 \leq a, b \leq r+4$, then we have

$$
\sum_{i=1}^{4} s a_{k-4, i} \bar{T}_{i a b}+\sum_{i=5}^{r+4} y_{i-4, k-4} \bar{T}_{i a b}=0 \quad \text { for } k \neq a, b .
$$

Proof. The proof follows from the fact that $\delta_{k a}=0$ and $\delta_{k b}=0$ for $k \neq p, q$.

Lemmas 3.7 and 3.8 enable us to define ideals associated with a skewsymmetrizable matrix $G_{3}$. These ideals contain a class of perfect ideals of grade 3 with type $2, \lambda=0$.

Definition 3.12. Using the notation given above, the alternating matrix $T=$ $\mathcal{A}\left(G_{3}\right)$ is obtained by multiplying the first four columns of $G_{3}$ by $s$. Let $x_{i}$ be the element defined by

$$
x_{i}=\bar{T}_{i} \quad \text { for } i=1,2,3, \ldots, r+4 .
$$

We define $\overline{P f_{r+3}\left(G_{3}\right)}$ as the ideal generated by $r+4$ elements $x_{i}$.

Remark 3.13. Let $G_{1}$ and $G_{2}$ be the $n \times n$ skew-symmetrizable matrices defined in (3.1) and (3.2), respectively. As we have shown that for $i=1,2$, the ideals $\overline{P f_{n-1}\left(G_{i}\right)}$ are linked to an almost complete intersection of grade 3 by a regular sequence $\mathbf{x}=x_{1}, x_{2}, x_{3}$. However we will see that the ideal $\overline{P f_{r+3}\left(G_{3}\right)}$ is not always linked to it by a regular sequence $\mathbf{x}$.

First we construct the minimal free resolution $\mathbb{F}$ of $R / \overline{P f_{r+3}\left(G_{3}\right)}$ such that the quotients of the maximal order Pfaffians of the submatrix $G_{3}$ of the second differential map in $\mathbb{F}$ generates a class of perfect ideals of grade 3 with type $2, \lambda=0$. Let $D_{n m u v}$ be the determinant of the $4 \times 4$ matrix formed by the four rows $n, m, u, v$ and columns $1,2,3,4$ of $A$. Define $W=\left(w_{i}\right)$ to be an $r \times 1$ matrix given by

$$
w_{i}=\sum_{1 \leq p<q \leq 4} \sum_{1 \leq u<v \leq r}\left|\begin{array}{ll}
a_{u p} & a_{u q} \\
a_{v p} & a_{v q}
\end{array}\right| Y_{i u v} U_{p q}+s \sum_{1 \leq a<b<c<d \leq r} Y_{i a b c d} D_{a b c d} .
$$

Now we construct the minimal free resolution $\mathbb{F}$ of $R / I$ such that $I$ is minimally generated by the quotients of the maximal order Pfaffians of the alternating matrix induced by the skew-symmetrizable submatrix $G_{3}$ of the second differential map in $\mathbb{F}$. Let $Z$ be $4 \times 1$ matrix given by

$$
Z=\left[\begin{array}{llll}
z_{1} & -z_{2} & z_{3} & -z_{4}
\end{array}\right]^{t}, \text { where } z_{i}=(-1)^{i} \sum_{l=1}^{r} Y_{l} a_{l i} .
$$


That is, $Z$ is the scalar multiplication of a matrix product of $A^{t}$ and the column vector $\left[\begin{array}{llll}Y_{1} & Y_{2} & \cdots & Y_{r}\end{array}\right]^{t}$ by -1 . Let $\mathbb{F}$ be the sequence of free $R$-modules and theirs maps defined by

$$
\mathbb{F}: 0 \longrightarrow R^{2} \stackrel{f_{3}}{\longrightarrow} R^{r+5} \stackrel{f_{2}}{\longrightarrow} R^{r+4} \stackrel{f_{1}}{\longrightarrow} R,
$$

where

$$
\begin{aligned}
f_{1} & =\left[\begin{array}{lllllllll}
x_{1} & x_{2} & x_{3} & x_{4} & x_{5} & x_{6} & x_{7} & \cdots & x_{r+4}
\end{array}\right] \\
f_{2} & =\left[\begin{array}{c|c|c|c|cccc|c}
U & s A^{t} & Z \\
\hline-A & Y & \mathbf{0}
\end{array}\right], \\
f_{3} & =\left[\begin{array}{cccc|ccccc|c}
0 & 0 & 0 & 0 & Y_{1} & Y_{2} & Y_{3} & \cdots & Y_{r} & s \\
\hline x_{1} & x_{2} & x_{3} & x_{4} & w_{1} & w_{2} & w_{3} & \cdots & w_{r} & -\operatorname{Pf}(U)
\end{array}\right]^{t} .
\end{aligned}
$$

First we show that $\mathbb{F}$ is a complex.

Lemma 3.14. Using the notation given above, let $G_{3 i}$ be the $(r+3) \times(r+3)$ submatrix of $G_{3}$ obtained by deleting the ith column and row from $G_{3}$. Then we have $f_{1} f_{2}=0$ and $f_{2} f_{3}=0$.

Proof. First we show that $f_{1} f_{2}=0$. It follows from part (2) of Lemma 2.1 that $f_{1} G_{3}=0$. Let $D_{g h l}^{(i)}$ is the determinant of the $3 \times 3$ matrix of $A$ formed by rows $g, h, l$ and columns $\alpha, \beta, \gamma$ of $A$ in this order and $\{i, \alpha, \beta, \gamma\}=\{1,2,3,4\}$. Simple computation shows that for $k=1,2,3,4$, we have

$$
\begin{aligned}
\sum_{k=1}^{4}(-1)^{k+1} x_{k} z_{k} & =\sum_{k=1}^{4} \sum_{1 \leq g<h<l \leq r} s D_{g h l}^{(k)} Y_{g h l} z_{k}+\sum_{k=1}^{4} \sum_{j=1}^{4}(-1)^{k+j+1} z_{k} z_{j} U_{k j} \\
& =\sum_{k=1}^{4} \sum_{1 \leq g<h<l \leq r}-s D_{g h l}^{(k)} Y_{g h l}(-1)^{k+1} \sum_{q=1}^{r} Y_{q} a_{q k} \\
& =\sum_{q=1}^{r} \sum_{1 \leq g<h<l \leq r}-s Y_{g h l}\left(\sum_{k=1}^{4}(-1)^{k+1} Y_{q} a_{q k} D_{g h l}^{(k)}\right) \\
& =\sum_{q=1}^{r} \sum_{1 \leq g<h<l \leq r}-s Y_{g h l} Y_{q} D_{q g h l} \\
& =\sum_{1 \leq a<b<c<d \leq r}-s\left(Y_{a} Y_{b c d}-Y_{b} Y_{a c d}+Y_{c} Y_{a b d}-Y_{d} Y_{a b c}\right) D_{a b c d}=0 .
\end{aligned}
$$

The last identity follows from Lemma 2.3 and this says that $f_{1} f_{2}=0$. Now we prove that $f_{2} f_{3}=0$. It is sufficient to show that
(a) $s A^{t} \mathbf{y}^{t}+s Z=0$, where $\mathbf{y}=\left[\begin{array}{llll}Y_{1} & Y_{2} & \cdots & Y_{r}\end{array}\right]$,
(b) $U \mathbf{x}^{t}+s A^{t} W-P f(U) Z=0$, where $\mathbf{x}=\left[\begin{array}{llll}x_{1} & x_{2} & x_{3} & x_{4}\end{array}\right]$, 
(c) $-A \mathbf{x}^{t}+Y W=0$.

It is easy to show part (a). We prove part (b). The following computation gives us the proof:

$$
\begin{aligned}
\sum_{k=1}^{r} s a_{k i} w_{k}= & \sum_{k=1}^{r} \sum_{1 \leq p<q \leq 4} \sum_{1 \leq u<v \leq r} s a_{k i}\left|\begin{array}{ll}
a_{u p} & a_{u q} \\
a_{v p} & a_{v q}
\end{array}\right| Y_{k u v} U_{p q} \\
& +\sum_{k=1}^{r} \sum_{1 \leq a<b<c<d \leq r} s^{2} a_{k i} Y_{k a b c d} D_{a b c d} \\
= & \sum_{1 \leq p<q \leq 4} \sum_{1 \leq m<n<t \leq r} s D_{m n t}^{i p q} Y_{m n t} U_{p q} \\
& +\sum_{1 \leq e<f<g<h<l \leq r} s^{2} D_{e f g h l} Y_{\text {efghl }},
\end{aligned}
$$

where $D_{m n t}^{i p q}$ is the determinant of the $3 \times 3$ matrix of $A$ formed by rows $m, n, t$ and columns $i, p, q$ of $A$ in this order and $D_{\text {efghl }}$ is the determinant of the $5 \times 5$ matrix formed by the five rows $e, f, g, h, l$ and columns $1,2,3,4, i$ of $A$. Since $A$ is an $r \times 4$ matrix,

$$
\begin{aligned}
\sum_{k=1}^{r} s a_{k i} w_{k} & =\sum_{1 \leq p<q \leq 4} \sum_{1 \leq m<n<t \leq r} s D_{m n t}^{i p q} Y_{m n t} U_{p q} \\
& =\sum_{h=1}^{4} \sum_{1 \leq u<v \leq 4} \sum_{1 \leq m<n<t \leq r} s D_{m n t}^{i u v} Y_{m n t} u_{h i} U_{h i u v}
\end{aligned}
$$

where $u<v$ in $\{h, i, u, v\}=\{1,2,3,4\}$. Hence for each $i$, we have

$$
\begin{aligned}
& \sum_{k=1}^{4} u_{i k} x_{k}+\sum_{k=1}^{r} s a_{k i} w_{k}+(-1)^{i+1}(-P f(U)) z_{i} \\
= & \sum_{k=1}^{4} \sum_{1 \leq g<h<l \leq r}(-1)^{k+1} s D_{g h l}^{(k)} Y_{g h l} u_{i k} \\
& -\sum_{k=1}^{4} \sum_{j=1}^{4}(-1)^{j+1} z_{j} u_{i k} U_{k j}+\sum_{k=1}^{r} s a_{k i} w_{k}+(-1)^{i+1}(-P f(U)) z_{i} \\
= & \sum_{k=1}^{4} \sum_{1 \leq g<h<l \leq r}(-1)^{k+1} s D_{g h l}^{(k)} Y_{g h l} u_{i k}+\sum_{k=1}^{r} s a_{k i} w_{k} \\
= & \sum_{k=1}^{4} \sum_{1 \leq g<h<l \leq r}(-1)^{k+1} s D_{g h l}^{(k)} Y_{g h l} u_{i k} \\
& +\sum_{h=1}^{4} \sum_{1 \leq u<v \leq 4} \sum_{1 \leq m<n<t \leq r} s D_{m n t}^{i u v} Y_{m n t} u_{h i} U_{h i u v}=0 .
\end{aligned}
$$


The second identity follows from part (1) of Lemma 2.1. Finally we show part (c).

For each $i$,

$$
\begin{aligned}
& \sum_{k=1}^{4}-a_{i k} x_{k}+\sum_{k=1}^{r} y_{i k} w_{k} \\
= & \sum_{k=1}^{4} \sum_{1 \leq g<h<l \leq r}(-1)^{k} s a_{i k} D_{g h l}^{(k)} Y_{g h l}-\sum_{k=1}^{4} \sum_{j=1}^{4} \sum_{l=1}^{r} a_{i k} a_{l j} Y_{l} U_{k j} \\
& +\sum_{k=1}^{r} \sum_{1 \leq p<q \leq 4} \sum_{1 \leq u<v \leq r} y_{i k}\left|\begin{array}{ll}
a_{u p} & a_{u q} \\
a_{v p} & a_{v q}
\end{array}\right| Y_{k u v} U_{p q} \\
& +\sum_{k=1}^{r} \sum_{1 \leq a<b<c<d \leq r} s y_{i k} Y_{k a b c d} D_{a b c d} \\
= & -\sum_{k=1}^{4} \sum_{j=1}^{4} \sum_{l=1}^{r} a_{i k} a_{l j} Y_{l} U_{k j}+\sum_{k=1}^{r} \sum_{1 \leq p<q \leq 4} \sum_{1 \leq u<v \leq r} y_{i k}\left|\begin{array}{ll}
a_{u p} & a_{u q} \\
a_{v p} & a_{v q}
\end{array}\right| Y_{k u v} U_{p q}=0 .
\end{aligned}
$$

The second identity follows from part (3) of Lemma 2.2. The last identity follows from part (1) of Lemma 2.2 and the following identities:

$$
\begin{aligned}
& \sum_{k=1}^{4} \sum_{j=1}^{4} \sum_{l=1}^{r} a_{i k} a_{l j} Y_{l} U_{k j}=\sum_{l=1}^{r} \sum_{1 \leq p<q \leq 4}\left|\begin{array}{ll}
a_{i p} & a_{i q} \\
a_{l p} & a_{l q}
\end{array}\right| Y_{l} U_{p q} \quad \text { and } \\
& \sum_{k=1}^{r} \sum_{1 \leq p<q \leq 4} \sum_{1 \leq u<v \leq r} y_{i k}\left|\begin{array}{ll}
a_{u p} & a_{u q} \\
a_{v p} & a_{v q}
\end{array}\right| Y_{k u v} U_{p q}=\sum_{h=1}^{r} \sum_{1 \leq p<q \leq 4}\left|\begin{array}{ll}
a_{i p} & a_{i q} \\
a_{h p} & a_{h q}
\end{array}\right| Y_{h} U_{p q} .
\end{aligned}
$$

The following proposition gives us a criterion which tests wether or not a perfect ideal $I$ of grade 3 with 2 has a property that $\lambda(I)=0$.

Proposition 3.15 ([Proposition 2.5, 2]). Let $R$ be a Noetherian local ring with maximal ideal $\mathfrak{m}$. Let $I$ be a grade 3 , type 2 perfect ideal. If $I$ is minimally generated by at least 5 elements, then the following are equivalent:

(1) $\lambda(I)>0$.

(2) There is a minimal set of generators $x_{1}, \ldots, x_{n}$ for $I$ such that $\mathbf{x}=$ $x_{1}, x_{2}, x_{3}$ is a regular sequence and $(\mathbf{x}): I$ is an almost complete intersection.

Example 3.16. Let $R=\mathbb{C}[[x, y, z]]$ be a formal power series ring over the field $\mathbb{C}$ of complex numbers with indeterminates $x, y, z$. If $I=\left(x^{2}, y^{2}, y z^{2}, x z^{2}, z^{3}\right)$ is an ideal, then $I$ has grade 3 and $x^{2}, y^{2}$ and $z^{3}$ are a regular sequence in $I$. Also we can easily show that type $I=2$ by finding the minimal free resolution $\mathbb{F}$ of $R / I$. The minimal free resolution $\mathbb{F}$ of $R / I$ is

$$
\mathbb{F}: 0 \longrightarrow R^{2} \stackrel{d_{3}}{\longrightarrow} R^{6} \stackrel{d_{2}}{\longrightarrow} R^{5} \stackrel{d_{1}}{\longrightarrow} R,
$$


where

$$
\begin{aligned}
d_{1}= & {\left[\begin{array}{lllll}
x^{2} & y^{2} & y z^{2} & x z^{2} & z^{3}
\end{array}\right], } \\
d_{2}= & {\left[\begin{array}{cccccc}
0 & 0 & 0 & 0 & z^{2} & y^{2} \\
0 & 0 & z^{2} & 0 & 0 & -x^{2} \\
z & 0 & -y & x & 0 & 0 \\
0 & z & 0 & -y & -x & 0 \\
-y & -x & 0 & 0 & 0 & 0
\end{array}\right], d_{3}=\left[\begin{array}{cc}
x & 0 \\
-y & 0 \\
0 & -x^{2} \\
-z & -x y \\
0 & y^{2} \\
0 & -z^{2}
\end{array}\right] . }
\end{aligned}
$$

And $L=K: I=\left(z, x y, y^{2}, x^{2}\right)$, where $K=\left(x^{2}, y^{2}, z^{3}\right)$. Since $x^{2}, y^{2}$ and $z$ are a regular sequence in $L$, it is an almost complete intersection of grade 3 . By Proposition 3.15, $\lambda(I)>0$.

The following theorem says that if $\overline{P f_{r+3}\left(G_{3}\right)}$ has grade 3 , then it has type 2 .

Theorem 3.17. Let $R$ be a Noetherian local ring with maximal ideal $\mathfrak{m}$. Let $r$ be an odd integer with $r>1$ and let $s$ be a regular element in $R$. With the notations above, we assume that the entries of $A, U$, and $Y$ are contained in $\mathfrak{m}$. Let $G_{3}$ be the $(r+4) \times(r+4)$ skew-symmetrizable matrix defined in (3.3). If $I=\overline{P f_{r+3}\left(G_{3}\right)}$ has grade 3 , then it is a perfect ideal of type 2 .

Proof. Let $\mathbb{F}$ be the complex of free $R$-modules and $R$-maps defined in (3.5). We prove that $\mathbb{F}$ is the minimal free resolution of $R / I$. We use the BuchsbaumEisenbud acyclicity criterion [3] to show that $\mathbb{F}$ is exact. It is easy to show that $f_{2}$ and $f_{3}$ have ranks $r+3$ and 2 , respectively. Hence the first condition of the criterion is satisfied. Now we prove that the second condition of it is also satisfied. Let $G_{3 i}$ be the $(r+3) \times(r+3)$ submatrix of $G_{3}$ obtained by deleting the $i$ th column and row from $G_{3}$. It follows from parts (1) and (2) of Lemma 3.7 that $\operatorname{det}\left(G_{3 i}\right)=s x_{i}^{2}$ for $i=1,2,3,4$. More precisely,

$$
\begin{aligned}
x_{i}^{3} & =\left(-\sum_{j=1}^{4}(-1)^{j+1} z_{j} U_{i j}+s \sum_{1 \leq g<h<l \leq r}(-1)^{i+1} D_{g h l}^{(i)} Y_{g h l}\right)^{3} \\
& =\left(-\sum_{j=1}^{4}(-1)^{j+1} z_{j} U_{i j}+s \sum_{1 \leq g<h<l \leq r}(-1)^{i+1} D_{g h l}^{(i)} Y_{g h l}\right) x_{i}^{2} \\
& =\sum_{j=1}^{4} \sum_{l=1}^{r} Y_{l} a_{l j} U_{i j} \times x_{i}^{2}+\sum_{1 \leq g<h<l \leq r}(-1)^{i+1} D_{g h l}^{(i)} Y_{g h l} \times s x_{i}^{2} \\
& =\sum_{j=1}^{4} \sum_{l=1}^{r} a_{l j} U_{i j} Y_{l} \times x_{i}^{2}+\sum_{1 \leq g<h<l \leq r}(-1)^{i+1} D_{g h l}^{(i)} Y_{g h l} \times \operatorname{det}\left(G_{3 i}\right) .
\end{aligned}
$$


Let $G_{i}^{i, l+4}$ be the $(r+3) \times(r+3)$ submatrix of $f_{2}$ obtained by deleting the row $i$ and two columns $i, l+4$ from $f_{2}$. Since

$$
\begin{aligned}
& s \times\left(\sum_{j=1}^{4} \sum_{l=1}^{r} a_{l j} U_{i j} Y_{l} x_{i}^{2}\right) \\
= & \sum_{j=1}^{4} \sum_{l=1}^{r} a_{l j} U_{i j} Y_{l} \operatorname{det}\left(G_{3 i}\right) \\
= & \sum_{j=1}^{4} a_{1 j} U_{i j} Y_{1} \operatorname{det}\left(G_{3 i}\right)+\sum_{j=1}^{4} a_{2 j} U_{i j} Y_{2} \operatorname{det}\left(G_{3 i}\right)+\cdots+\sum_{j=1}^{4} a_{r j} U_{i j} Y_{r} \operatorname{det}\left(G_{3 i}\right)
\end{aligned}
$$

and

$$
Y_{l} \operatorname{det}\left(G_{3 i}\right)=(-1)^{l} s \operatorname{det}\left(G_{i}^{i, l+4}\right),
$$

we have

$$
\sum_{j=1}^{4} \sum_{l=1}^{r} a_{l j} U_{i j} Y_{l} x_{i}^{2} \in\left(\operatorname{det}\left(G_{i}^{i, 5}\right), \operatorname{det}\left(G_{i}^{i, 6}\right), \operatorname{det}\left(G_{i}^{i, 7}\right), \ldots, \operatorname{det}\left(G_{i}^{i, r+4}\right)\right)
$$

for $i=1,2,3,4$.

Thus

$$
\sum_{j=1}^{4} \sum_{l=1}^{r} a_{l j} U_{i j} Y_{l} x_{i}^{2} \in I_{r+3}\left(f_{2}\right) .
$$

Hence $x_{i}^{3} \in I_{r+3}\left(f_{2}\right)$ for $i=1,2,3,4$. Part (3) of Lemma 3.7 says that for $i=5,6, \ldots, r+4$,

$$
\operatorname{det} G_{3 i}=\mathcal{A}\left(G_{3}\right)_{i}^{2} / s^{4}=x_{i}^{2} \text { implies } x_{i}^{2} \text { is in } I_{r+3}\left(f_{2}\right) \text {. }
$$

The fact that $x_{i}^{2}$ is contained in $I_{2}\left(f_{3}\right)$ can be proved as follows: For $i=1,2,3,4$,

$$
\begin{aligned}
x_{i}^{2} & =\left(-\sum_{j=1}^{4}(-1)^{j+1}(-1)^{j} \sum_{l=1}^{r} Y_{l} a_{l j} U_{i j}+s \sum_{1 \leq g<h<l \leq r}(-1)^{i+1} D_{g h l}^{(i)} Y_{g h l}\right) x_{i} \\
& =\sum_{j=1}^{4} \sum_{l=1}^{r} Y_{l} x_{i} a_{l j} U_{i j}+s x_{i} \sum_{1 \leq g<h<l \leq r}(-1)^{i+1} D_{g h l}^{(i)} Y_{g h l} \in I_{2}\left(f_{3}\right) .
\end{aligned}
$$

And for $i=5,6, \ldots, r+4$,

$$
x_{i}=-\left(Y_{i-4}(-P f(U))-s w_{i-4}\right) \text { implies that } x_{i} \text { is in } I_{2}\left(f_{3}\right) .
$$

Thus if $I$ has grade 3 , then it has type 2 .

Example 3.18. Let $R=\mathbb{Q}[x, y, z]$ be the polynomial ring over the field $\mathbb{Q}$ of rationals with indeterminates $x, y, z$ and $\operatorname{deg} x=\operatorname{deg} y=\operatorname{deg} z=1$. Let $A, Y$, 
and $U$ be a $3 \times 4$ matrix, a $3 \times 3$ alternating matrix, and $4 \times 4$ matrix given by

$$
A=\left[\begin{array}{llll}
0 & y & x & x \\
y & 0 & 0 & 0 \\
z & y & 0 & z
\end{array}\right], Y=\left[\begin{array}{ccc}
0 & z^{2} & y^{2} \\
-z^{2} & 0 & x^{2} \\
-y^{2} & -x^{2} & 0
\end{array}\right], \text { and } U=\left[\begin{array}{cccc}
0 & x & z & 0 \\
-x & 0 & z & x \\
-z & -z & 0 & y \\
0 & -x & -y & 0
\end{array}\right],
$$

respectively. Let $s=y$. Let $G_{3}$ be the matrix defined in (3.3). Then the $7 \times 7$ alternating matrix $\mathcal{A}\left(G_{3}\right)$ has following form

$$
\begin{aligned}
\mathcal{A}\left(G_{3}\right)= & {\left[\begin{array}{c|c}
s U & s A^{t} \\
\hline-s A & Y
\end{array}\right] } \\
= & {\left[\begin{array}{cccc|ccc}
0 & x y & y z & 0 & 0 & y^{2} & y z \\
-x y & 0 & y z & x y & y^{2} & 0 & y^{2} \\
-y z & -y z & 0 & y^{2} & x y & 0 & 0 \\
0 & -x y & -y^{2} & 0 & x y & 0 & y z \\
\hline 0 & -y^{2} & -x y & -x y & 0 & z^{2} & y^{2} \\
-y^{2} & 0 & 0 & 0 & -z^{2} & 0 & x^{2} \\
-y z & -y^{2} & 0 & -y z & -y^{2} & -x^{2} & 0
\end{array}\right] }
\end{aligned}
$$

and then $I=\overline{P f_{6}\left(G_{3}\right)}$ is an ideal generated by the following seven elements

$$
\begin{aligned}
& x_{1}=-x^{4}+x^{2} y^{2}+x^{3} z+y^{2} z^{2}+z^{4}, \\
& x_{2}=y^{4}-x^{3} z-x y^{2} z-y z^{3}-z^{4}, \\
& x_{3}=x^{4}-2 x y^{3}+y^{3} z+2 x z^{3}, \\
& x_{4}=-x^{4}+x y^{3}+x^{2} y z+y^{3} z+y z^{3}-z^{4}, \\
& x_{5}=x^{3} y-y^{4}-x^{3} z-y^{2} z^{2}, \\
& x_{6}=-x y^{3}+2 x^{2} y z+2 x y^{2} z-y^{3} z-x y z^{2}-y^{2} z^{2}, \\
& x_{7}=-x^{2} y^{2}+y^{4}+x y^{2} z+x y z^{2}-x z^{3} .
\end{aligned}
$$

Now we will show that $I$ is a perfect ideal of grade 3 with type 2 and $\lambda=0$. First we show that $I$ has grade 3 . Using CoCoA 4.7.4, we can easily check that the radical of $I$ is the maximal ideal $\mathfrak{m}=(x, y, z)$. Since $\mathfrak{m}$ is the prime ideal of grade 3, it follows that $I$ has grade 3. Easy computations by CoCoA 4.7.4 gives us that

$$
\begin{aligned}
z_{1} & =y^{3}-z^{3}, \quad z_{2}=x^{2} y+y z^{2}, z_{3}=-x^{3}, z_{4}=x^{3}+z^{3}, \\
w_{1} & =y^{3}+y z^{2}, \quad w_{2}=-2 x^{2} z-x y z+y^{2} z+x z^{2}+y z^{2}, \quad w_{3}=x^{2} y-y^{3}-x y z .
\end{aligned}
$$

The minimal free resolution $\mathbb{F}$ of $R / \overline{P f_{6}\left(G_{3}\right)}$ has the form:

$$
\mathbb{F}: 0 \longrightarrow R^{2} \stackrel{f_{3}}{\longrightarrow} R^{8} \stackrel{f_{2}}{\longrightarrow} R^{7} \stackrel{f_{1}}{\longrightarrow} R
$$

where

$$
f_{1}=\left[\begin{array}{lllllll}
x_{1} & x_{2} & x_{3} & x_{4} & x_{5} & x_{6} & x_{7}
\end{array}\right],
$$




$$
\begin{aligned}
& f_{2}=\left[\begin{array}{c|c|c}
U & s A^{t} & Z \\
\hline-A & Y & \mathbf{0}
\end{array}\right] \\
& =\left[\begin{array}{cccc|ccc|c}
0 & x & z & 0 & 0 & y^{2} & y z & z_{1} \\
-x & 0 & z & x & y^{2} & 0 & y^{2} & -z_{2} \\
-z & -z & 0 & y & x y & 0 & 0 & z_{3} \\
0 & -x & -y & 0 & x y & 0 & y z & -z_{4} \\
\hline 0 & -y & -x & -x & 0 & z^{2} & y^{2} & 0 \\
-y & 0 & 0 & 0 & -z^{2} & 0 & x^{2} & 0 \\
-z & -y & 0 & -z & -y^{2} & -x^{2} & 0 & 0
\end{array}\right] \text {, } \\
& f_{3}=\left[\begin{array}{c|c}
0 & \mathbf{q}^{t} \\
\hline \mathbf{y}^{t} & W \\
\hline s & -P f(U)
\end{array}\right] \\
& =\left[\begin{array}{cccc|ccc|c}
0 & 0 & 0 & 0 & Y_{1} & Y_{2} & Y_{3} & x \\
\hline x_{1} & x_{2} & x_{3} & x_{4} & w_{1} & w_{2} & w_{3} & -y^{2}
\end{array}\right]^{t}, \\
& \mathbf{q}=\left[\begin{array}{llll}
x_{1} & x_{2} & x_{3} & x_{4}
\end{array}\right] .
\end{aligned}
$$

This shows that $I$ has type 2 . Finally we prove that $\lambda(I)=0$. We can prove this by Proposition 3.15 as follows. Easy computation by CoCoA 4.7.4 shows that $\mathbf{x}=x_{1}, x_{2}, x_{3}$ is a regular sequence. Let $J=(\mathbf{x}): I$. Then by Theorem $2.6 J$ is a perfect ideal of grade 3. The minimal free resolution $\mathbb{G}$ of $R / J$ is

$$
\mathbb{G}: 0 \longrightarrow R^{4} \longrightarrow R^{8} \longrightarrow R^{5} \longrightarrow R \text {. }
$$

Hence $J$ is a perfect ideal of grade 3 minimally generated by five elements. Since $I$ is minimally generated by $r+4$ elements, it follows from the Bass's result [1] that the type of $J$ is $r+1$. Let $\mathbf{x}=x_{i}, x_{j}, x_{k}$ be any regular sequence in $I$ and $J=(\mathbf{x}): I$. Then we can easily check by CoCoA 4.7.4 that by finding the minimal free resolution of $R / J, J$ is a perfect ideal of grade 3 minimally generated by five elements. Actually the minimal free resolution of $R / J$ has the form defined in (3.6). Proposition 3.15 gives us that $\lambda(I)=0$.

\section{Hilbert functions of some classes of perfect ideals of grade 3}

Let $R=k\left[v_{0}, v_{1}, \ldots, v_{m}\right]$ be the polynomial ring over the field $k$ with indeterminates $v_{i}$ and $\operatorname{deg} v_{i}=1$. Let $I$ be a homogeneous Gorenstein ideal of grade 3 . For this purpose, we use the explicit description of minimal free resolution for $R / I$ in [2] to describe the Hilbert function of $R / I$. Consider the minimal free resolution $\mathbb{F}$ in the form

$$
\mathbb{F}_{\text {hom }}: 0 \longrightarrow R(-s) \stackrel{f_{1}^{*}}{\longrightarrow} \bigoplus_{i=1}^{n} R\left(-p_{i}\right) \stackrel{f_{2}}{\longrightarrow} \bigoplus_{i=1}^{n} R\left(-q_{i}\right) \stackrel{f_{1}}{\longrightarrow} R(0),
$$

where $f_{i}$ is a homogeneous map of degree 0 for $i=1,2,3$, and $f_{1}=\left(y_{i}\right)$, $f_{2}=\left(f_{i j}\right)$,

$$
q_{i}=\operatorname{deg} y_{i}, \quad p_{j}=\operatorname{deg} f_{i j}+q_{i}, \quad s=p_{i}+q_{i}
$$


Of course, we may also have $f_{i j}=0$ when $i=j$. If we define $r_{i}=p_{i}-q_{i}$ and use the fact that $y_{i}$ is a certain pfaffian of $f_{2}$, we get

$$
\operatorname{deg} y_{i}=\frac{s-r_{i}}{2}, \operatorname{deg} f_{i j}=\frac{r_{i}+r_{j}}{2}, \text { and } s=\sum_{i=1}^{n} r_{i}
$$

Using the original method for computing the Hilbert function of $R / I[7]$, we get the following proposition.

Proposition 4.1 ([Proposition 3.3, 4]). Using the notation given above we have

$H(R / I, t)=\left(\begin{array}{c}m+t \\ m\end{array}\right)-\sum_{i=1}^{n}\left(\begin{array}{c}m+t-q_{i} \\ m\end{array}\right)+\sum_{i=1}^{n}\left(\begin{array}{c}m+t-p_{i} \\ m\end{array}\right)-\left(\begin{array}{c}m+t-s \\ m\end{array}\right)$.

Let $I$ be the homogeneous perfect ideal of grade 3 with type 2 defined in section 3. Now we describe the Hilbert function of $R / I$. We can rewrite the minimal free resolution of $R / I$ in (3.5) in the following form

$$
\mathbb{F}_{\text {hom }}: 0 \longrightarrow \bigoplus_{i=1}^{2} R\left(s_{i}\right) \stackrel{f_{3}}{\longrightarrow} \bigoplus_{i=1}^{r+5} R\left(-p_{i}\right) \stackrel{f_{2}}{\longrightarrow} \bigoplus_{i=1}^{r+4} R\left(-q_{i}\right) \stackrel{f_{1}}{\longrightarrow} R(0)
$$

where $f_{i}$ are defined in Section 3. The degrees $q_{i}$ of the homogeneous generators for the ideal and the shifted degrees $p_{i}$ are given by

$$
\begin{aligned}
& q_{i}=\operatorname{deg} x_{i} \text { for } i=1,2, \ldots, r+4, \\
& p_{1}=\operatorname{deg} u_{21}+q_{2} \text { or } p_{1}=\operatorname{deg} u_{31}+q_{3} \text { or } p_{1}=\operatorname{deg} u_{41}+q_{4} \text { or } \\
& p_{1}=\operatorname{deg} a_{l 1}+q_{l+4} \text { for some } l(1 \leq l \leq r) \\
& p_{2}=\operatorname{deg} u_{12}+q_{1} \text { or } p_{2}=\operatorname{deg} u_{32}+q_{3} \text { or } p_{2}=\operatorname{deg} u_{42}+q_{4} \text { or } \\
& p_{2}=\operatorname{deg} a_{l 2}+q_{l+4} \text { for some } l(1 \leq l \leq r) \\
& p_{3}=\operatorname{deg} u_{13}+q_{1} \text { or } p_{3}=\operatorname{deg} u_{23}+q_{2} \text { or } p_{3}=\operatorname{deg} u_{43}+q_{4} \text { or } \\
& p_{3}=\operatorname{deg} a_{l 3}+q_{l+4} \text { for some } l(1 \leq l \leq r) \\
& p_{4}=\operatorname{deg} u_{14}+q_{1} \text { or } p_{4}=\operatorname{deg} u_{24}+q_{2} \text { or } p_{4}=\operatorname{deg} u_{34}+q_{3} \text { or } \\
& p_{4}=\operatorname{deg} a_{l 4}+q_{l+4} \text { for some } l(1 \leq l \leq r), \\
& p_{i}=\operatorname{deg} s+\operatorname{deg} a_{i-4, l}+q_{l} \text { or } p_{i}=\operatorname{deg} y_{c-4, i-4}+q_{c} \text { for } i=5,6, \ldots, r+4, \\
& \quad \text { for some } l(1 \leq l \leq 4) \text { and } c \text { is an integer with } 5 \leq c \leq r+4, \\
& p_{t}=\operatorname{deg} z_{i}+q_{i} \text { for some } i(1 \leq i \leq 4) \text { and } t=r+5, \\
& s_{1}=\operatorname{deg} Y_{k}+p_{k+4} \text { for some } k(1 \leq k \leq r) \text { or } s_{1}=\operatorname{deg} s+p_{r+5}, \\
& s_{2}=\operatorname{deg} x_{l}+p_{l} \text { for some } l(1 \leq l \leq 4) \text { or } s_{2}=\operatorname{deg} w_{k}+p_{k+4} \\
& \quad \text { for some } k(1 \leq k \leq r) \text { or } s_{2}=\operatorname{deg} \operatorname{Pf}(U)+p_{r+5} .
\end{aligned}
$$

In the same way mentioned above we can compute the Hilbert function of $R / I$ as follows. 
Proposition 4.2. With notations as above we have

$H(R / I, t)=\left(\begin{array}{c}m+t \\ m\end{array}\right)-\sum_{i=1}^{r+4}\left(\begin{array}{c}m+t-q_{i} \\ m\end{array}\right)+\sum_{i=1}^{r+5}\left(\begin{array}{c}m+t-p_{i} \\ m\end{array}\right)-\sum_{i=1}^{2}\left(\begin{array}{c}m+t-s_{i} \\ m\end{array}\right)$.

Here is an example.

Example 4.3. Let $R=\mathbb{C}[x, y, z]$ be the polynomial ring over the field $\mathbb{C}$ of complex numbers with indeterminates $x, y, z$ and $\operatorname{deg} x=\operatorname{deg} y=\operatorname{deg} z=1$. Let

$$
A=\left[\begin{array}{cccc}
-y & 0 & -x & 0 \\
0 & -z & 0 & 0 \\
-z & 0 & -y & 0
\end{array}\right], Y=\left[\begin{array}{ccc}
0 & y^{2} & z^{2} \\
-y^{2} & 0 & x^{2} \\
-z^{2} & -x^{2} & 0
\end{array}\right], U=\left[\begin{array}{cccc}
0 & x & z & y \\
-x & 0 & y & x \\
-z & -y & 0 & z \\
-y & -x & -z & 0
\end{array}\right] \text {. }
$$

Let $s=z$. Then the $7 \times 7$ skew-symmetrizable matrix $G_{3}$ is of the form

$$
G_{3}=\left[\begin{array}{c|c}
U & s A^{t} \\
\hline-A & Y
\end{array}\right]
$$

and the alternating matrix induced by $G_{3}$ is

$$
\begin{aligned}
& \mathcal{A}\left(G_{3}\right)=\left[\begin{array}{c|c}
s U & s A^{t} \\
\hline-s A & Y
\end{array}\right] \\
& =\left[\begin{array}{cccc|ccc}
0 & x z & z^{2} & y z & -y z & 0 & -z^{2} \\
-x z & 0 & y z & x z & 0 & -z^{2} & 0 \\
-z^{2} & -y z & 0 & z^{2} & -x z & 0 & -y z \\
-y z & -x z & -z^{2} & 0 & 0 & 0 & 0 \\
\hline y z & 0 & x z & 0 & 0 & y^{2} & z^{2} \\
0 & z^{2} & 0 & 0 & -y^{2} & 0 & x^{2} \\
z^{2} & 0 & y z & 0 & -z^{2} & -x^{2} & 0
\end{array}\right]
\end{aligned}
$$

and thus $I=\overline{P f_{6}\left(\mathcal{A}\left(G_{3}\right)\right)}$ is the ideal generated by seven homogeneous elements

$$
\begin{aligned}
& x_{1}=x^{4}+x y^{3}+z^{4}, \\
& x_{2}=-x^{3} y-y^{4}+x^{2} y z+y^{2} z^{2}, \\
& x_{3}=-x^{3} y-x y^{2} z-y z^{3}, \\
& x_{4}=x^{4}+x^{2} y^{2}+x y^{3}+y^{3} z-y^{2} z^{2}+x z^{3}+z^{4}, \\
& x_{5}=x^{2} y^{2}-y^{2} z^{2}+z^{4}, \\
& x_{6}=-x y^{2} z+x^{2} z^{2}-y^{2} z^{2}, \\
& x_{7}=y^{4}+x y z^{2}-y z^{3} .
\end{aligned}
$$

Now we show that $I$ is a homogeneous perfect ideal of grade 3 with type 2 . Easy computation by CoCoA 4.7.4 says that the radical of $I$ is the maximal 
ideal $\mathfrak{m}=(x, y, z)$. Since $\mathfrak{m}$ has grade $3, I$ has grade 3 . The minimal free resolution $\mathbb{F}$ of $R / I$ is given by (3.5).

$$
\mathbb{F}: 0 \longrightarrow \bigoplus_{i=i}^{2} R\left(s_{i}\right) \stackrel{f_{3}}{\longrightarrow} \bigoplus_{i=1}^{8} R\left(-p_{i}\right) \stackrel{f_{2}}{\longrightarrow} \bigoplus_{i=1}^{7} R\left(-q_{i}\right) \stackrel{f_{1}}{\longrightarrow} R(0),
$$

where

$$
\begin{aligned}
& f_{1}=\left[\begin{array}{lllllll}
x_{1} & x_{2} & x_{3} & x_{4} & x_{5} & x_{6} & x_{7}
\end{array}\right], \\
& f_{2}=\left[\begin{array}{c|c|c}
U & s A^{t} & Z \\
\hline-A & Y & \mathbf{0}
\end{array}\right] \\
& =\left[\begin{array}{cccc|ccc|c}
0 & x & z & y & -y z & 0 & -z^{2} & x^{2} y+y^{2} z \\
-x & 0 & y & x & 0 & -z^{2} & 0 & -z^{3} \\
-z & -y & 0 & z & -x z & 0 & -y z & x^{3}+y^{3} \\
-y & -x & -z & 0 & 0 & 0 & 0 & 0 \\
\hline y & 0 & x & 0 & 0 & y^{2} & z^{2} & 0 \\
0 & z & 0 & 0 & -y^{2} & 0 & x^{2} & 0 \\
z & 0 & y & 0 & -z^{2} & -x^{2} & 0 & 0
\end{array}\right], \\
& f_{3}=\left[\begin{array}{c|c}
0 & \mathbf{q}^{t} \\
\hline \mathbf{y}^{t} & W \\
\hline s & -P f(U)
\end{array}\right] \\
& =\left[\begin{array}{cccc|ccc|c}
0 & 0 & 0 & 0 & Y_{1} & Y_{2} & Y_{3} & z \\
\hline x_{1} & x_{2} & x_{3} & x_{4} & w_{1} & w_{2} & w_{3} & -y^{2}
\end{array}\right]^{t}, \\
& \mathbf{q}=\left[\begin{array}{l}
x_{1} \\
x_{2} \\
x_{3} \\
x_{4}
\end{array}\right]^{t}
\end{aligned}
$$

and

$Y_{1}=x^{2}, Y_{2}=-z^{2}, Y_{3}=y^{2}, w_{1}=z^{3}-y^{2} z, w_{2}=x^{2} z-x y^{2}, w_{3}=x y z-y z^{2}$.

Hence $I$ is a homogeneous perfect ideal of grade 3 with type 2 . Now we compute the Hilbert function $R / I$ by using Proposition 4.2. The values of the $p_{i}, q_{i}$ and $s_{i}$ are given as follows

$$
\begin{aligned}
& q_{i}=4 \text { for } i=1,2, \ldots, 7, \\
& p_{i}=5 \text { for } i=1,2,3,4, \\
& p_{j}=6 \text { for } j=5,6,7, \\
& p_{8}=7, \quad s_{1}=8, \quad s_{2}=9 .
\end{aligned}
$$

Hence the Hilbert function $H(R / I, t)$ is as follows:

$$
\begin{aligned}
& H(R / I, 0)=1, H(R / I, 1)=3, H(R / I, 2)=6, H(R / I, 3)=10, \\
& H(R / I, 4)=8, H(R / I, 5)=4, H(R / I, 6)=1, H(R / I, 7)=0 \text { for } t \geq 7 .
\end{aligned}
$$


This agrees with the computation by CoCoA 4.7.4.

Acknowledgements. We would like to thank anonymous referees for giving us valuable suggestions and constructive criticism.

\section{References}

[1] H. Bass, On the ubiquity of Gorenstein rings, Math. Z. 82 (1963), 8-28.

[2] A. Brown, A structure theorem for a class of grade three perfect ideals, J. Algebra 105 (1987), no. 2, 308-327.

[3] D. A. Buchsbaum and D. Eisenbud, What makes a complex exact?, J. Algebra 25 (1973), 259-268.

[4] - Algebra structures for finite free resolutions and some structure theorems for ideals of codimension 3, Amer. J. Math. 99 (1977), no. 3, 447-485.

[5] E. J. Choi, O.-J. Kang, and H. J. Ko, On the structure of the grade three perfect ideals of type three, Commun. Korean Math. Soc. 23 (2008), no. 4, 487-497.

[6] E. S. Golod, A note on perfect ideals, From the collection "Algebra" (A. I. Kostrikin, Ed), Moscow State Univ. Publishing House (1980), 37-39.

[7] D. Hilbert, Ueber die Theorie der Algebraischen Formen, Math. Ann. 36 (1890), no. 4, $473-534$.

[8] O.-J. Kang, Y. S. Cho, and H. J. Ko, Structure theory for some classes of grade three perfect ideals, J. Algebra 322 (2009), no. 8, 2680-2708.

[9] O.-J. Kang and H. J. Ko, The structure theorem for complete intersections of grade 4, Algebra Colloq. 12 (2005), no. 2, 181-197.

[10] Structure theorems for perfect ideals of grade g, Commun. Korean. Math. Soc. 21 (2006), no. 4, 613-630.

[11] A. Kustin and M. Miller, A general resolution for grade four Gorenstein ideals, Manuscripta Math. 35 (1981), no. 1-2, 221-269.

[12] - Structure theory for a class of grade four Gorenstein ideals, Trans. Amer. Math. Soc. 270 (1982), no. 1, 287-307.

[13] C. Peskine and L. Szpiro, Liaison des variétés algébriques, Invent. Math. 26 (1974), 271-302.

[14] R. Sanchez, Structure theorem for type 3, grade 3 perfect ideals, J. Algebra 123 (1989), no. 2, 263-288.

Yong Sung CHO

Department of Mathematics

YONSEI UNIVERSITY

SeOul 120-749, Korea

E-mail address: yongsung@yonsei.ac.kr

OH-JIN KANG

Department of Mathematics

UNIVERSITY OF INCHEON

INCHEON 402-749, KoREA

E-mail address: ohkang@incheon.ac.kr

Hyoung June Ko

Department of Mathematics

YONSEI UNIVERSITY

SEOUl 120-749, KoreA

E-mail address: hjko@yonsei.ac.kr 